\title{
Room-Temperature mobility above 2200 cm2/V.s of two-dimensional electron gas in a sharp-interface AlGaN/GaN heterostructure
}

Jr-Tai Chen, Ingemar Persson, Daniel Nilsson, Chih-Wei Hsu, Justinas Palisaitis, Urban

Forsberg, Per Persson and Erik Janzén

\section{Linköping University Post Print}

\section{Tweet}

N.B.: When citing this work, cite the original article.

Original Publication:

Jr-Tai Chen, Ingemar Persson, Daniel Nilsson, Chih-Wei Hsu, Justinas Palisaitis, Urban Forsberg, Per Persson and Erik Janzén, Room-Temperature mobility above $2200 \mathrm{~cm} 2 / \mathrm{V}$.s of two-dimensional electron gas in a sharp-interface AlGaN/GaN heterostructure, 2015, Applied Physics Letters, (106), 25.

http://dx.doi.org/10.1063/1.4922877

Copyright: American Institute of Physics (AIP) http://www.aip.org/

Postprint available at: Linköping University Electronic Press http://urn.kb.se/resolve?urn=urn:nbn:se:liu:diva-117133 


\section{AIP / Applied Physics

Room-temperature mobility above $2200 \mathrm{~cm} 2 / \mathrm{V} \cdot \mathrm{s}$ of two-dimensional electron gas in a sharp-interface AIGaN/GaN heterostructure

Jr-Tai Chen, Ingemar Persson, Daniel Nilsson, Chih-Wei Hsu, Justinas Palisaitis, Urban Forsberg, Per O. Å.

Persson, and Erik Janzén

Citation: Applied Physics Letters 106, 251601 (2015); doi: 10.1063/1.4922877

View online: http://dx.doi.org/10.1063/1.4922877

View Table of Contents: http://scitation.aip.org/content/aip/journal/apl/106/25?ver=pdfcov

Published by the AIP Publishing

\section{Articles you may be interested in}

Electronic transport characterization of $\mathrm{Al} \mathrm{Ga} \mathrm{N/Ga} \mathrm{N}$ heterostructures using quantitative mobility spectrum analysis

Appl. Phys. Lett. 91, 102113 (2007); 10.1063/1.2778453

Nanostructural characterization and two-dimensional electron-gas properties in high-mobility Al Ga N/Al N/Ga

$\mathrm{N}$ heterostructures grown on epitaxial AIN/sapphire templates

J. Appl. Phys. 98, 063713 (2005); 10.1063/1.2060946

Electron mobility exceeding $160000 \mathrm{~cm} 2 / \mathrm{V} \mathrm{s}$ in Al Ga N/Ga N heterostructures grown by molecular-beam epitaxy

Appl. Phys. Lett. 85, 5394 (2004); 10.1063/1.1824176

High-electron-mobility Al Ga N/AI N/Ga N heterostructures grown on 100 - mm -diam epitaxial AIN/sapphire templates by metalorganic vapor phase epitaxy

Appl. Phys. Lett. 85, 1710 (2004); 10.1063/1.1790073

AlGaN/GaN heterostructures on insulating AlGaN nucleation layers

Appl. Phys. Lett. 75, 388 (1999); 10.1063/1.124384

Frustrated by

old technology?

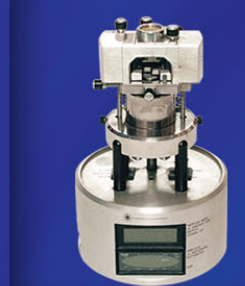

Is your AFM dead

and can't be repaired?

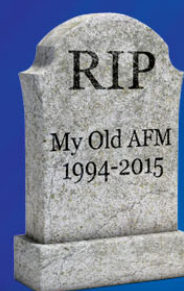

Sick of bad customer support?

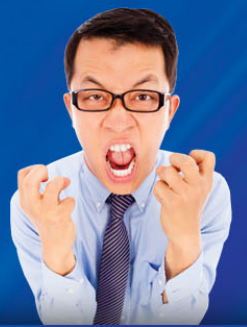

It is time to upgrade your AFM Minimum \$20,000 trade-in discount for purchases before August 31st

Asylum Research is today's technology leader in AFM 


\title{
Room-temperature mobility above $2200 \mathrm{~cm}^{2} / \mathrm{V} \cdot \mathrm{s}$ of two-dimensional electron gas in a sharp-interface AIGaN/GaN heterostructure
}

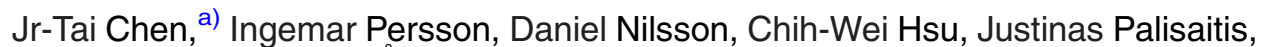 \\ Urban Forsberg, Per O. A. Persson, and Erik Janzén \\ Department of Physics, Chemistry, and Biology, Linköping University, SE 58183 Linköping, Sweden
}

(Received 14 April 2015; accepted 12 June 2015; published online 22 June 2015)

\begin{abstract}
A high mobility of $2250 \mathrm{~cm}^{2} / \mathrm{V} \cdot \mathrm{s}$ of a two-dimensional electron gas (2DEG) in a metalorganic chemical vapor deposition-grown $\mathrm{AlGaN} / \mathrm{GaN}$ heterostructure was demonstrated. The mobility enhancement was a result of better electron confinement due to a sharp $\mathrm{AlGaN} / \mathrm{GaN}$ interface, as confirmed by scanning transmission electron microscopy analysis, not owing to the formation of a traditional thin AlN exclusion layer. Moreover, we found that the electron mobility in the sharpinterface heterostructures can sustain above $2000 \mathrm{~cm}^{2} / \mathrm{V}$.s for a wide range of $2 \mathrm{DEG}$ densities. Finally, it is promising that the sharp-interface $\mathrm{AlGaN} / \mathrm{GaN}$ heterostructure would enable low contact resistance fabrication, less impurity-related scattering, and trapping than the AlGaN/AlN/GaN heterostructure, as the high-impurity-contained AIN is removed. ( 2015 AIP Publishing LLC.

[http://dx.doi.org/10.1063/1.4922877]
\end{abstract}

The transport properties of $\mathrm{AlGaN} / \mathrm{GaN}$ high electron mobility transistor (HEMT) structures have been studied intensively over the last two decades. In the high temperature regime $(>300 \mathrm{~K})$ of interest for most device applications, the mobility of the two-dimensional electron gas (2DEG) formed near the $\mathrm{AlGaN} / \mathrm{GaN}$ interface has been theoretically calculated and shown to be ultimately limited by phonon scattering. ${ }^{1,2}$ Nevertheless, in practice, other important scattering mechanisms related to structural imperfections of the materials including alloy disorder and interface roughness could actually influence the 2DEG mobility. Room-temperature 2DEG mobility in the $\mathrm{AlGaN} / \mathrm{GaN}$ heterostructure is typically reported in the range from 1300 to $1600 \mathrm{~cm}^{2} / \mathrm{V} \cdot \mathrm{s}$, depending on the 2DEG density and the $\mathrm{Al}$ content of the AlGaN barrier. ${ }^{3,4}$ By inserting a thin $(1-2 \mathrm{~nm})$ AlN exclusion layer $\left(\mathrm{AlN}_{\mathrm{ex}}\right)$ at the $\mathrm{AlGaN} / \mathrm{GaN}$ interface, the $2 \mathrm{DEG}$ mobility can be remarkably increased to $\sim 2200 \mathrm{~cm}^{2} / \mathrm{V} \cdot \mathrm{s} .{ }^{5-7}$ This mobility improvement is associated with better $2 \mathrm{DEG}$ confinement near the interface, reducing the penetration of the electron wave function into the $\mathrm{AlGaN}$ barrier, so that the scattering due to alloy disorder is alleviated. ${ }^{8}$ However, the presence of the thin $\mathrm{AlN}_{\mathrm{ex}}$ layer (or high-Al-content AlGaN layer) can raise the surface potential in a HEMT structure owing to its wide band gap nature, rendering difficulties in obtaining low ohmic contact resistance, ${ }^{9}$ which is essential for high-frequency applications. Additional recess etching into the $\mathrm{AlGaN}$ barrier for contact metallization processes becomes necessary to reduce the contact resistance. ${ }^{10,11}$ In this work, we show that the 2DEG mobility can be greatly improved in metalorganic chemical vapor deposition (MOCVD)-grown AlGaN/GaN heterostructures by sharpening the interface, without inserting an $\mathrm{AlN}_{\mathrm{ex}}$ layer. The effect of different $\mathrm{AlGaN} / \mathrm{GaN}$ interface structures on the 2DEG properties is presented, utilizing high-resolution scanning transmission electron microscopy (STEM).

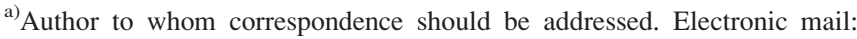
jrche@ifm.liu.se.
}

The AlGaN/GaN HEMT structures were grown on $2 \times 2 \mathrm{~cm}^{2} 4 \mathrm{H}$-semi-insulating (SI) $\mathrm{SiC}$ substrates in a hotwall MOCVD system at a pressure of 50 mbar in a mixture of $\mathrm{N}_{2}$ and $\mathrm{H}_{2}$ as the carrier gases. Details of the hot-wall MOCVD reactor and the complete HEMT structure growth process have been presented elsewhere., ${ }^{712}$ A series of AlGaN/GaN HEMT samples were grown to investigate the structural details in various $\mathrm{AlGaN} / \mathrm{GaN}$ interfaces and their effects on the 2DEG properties. The series consists of three $\sim 28$-nm-thick $\mathrm{Al}_{0.17} \mathrm{Ga}_{0.83} \mathrm{~N} / \mathrm{GaN}$ HEMT samples, denoted S1-S3. The growth conditions were identical for the three HEMT samples, except for the condition during the transition from the GaN layer to the $\mathrm{AlGaN}$ layer. Three different interface structures of the $\mathrm{AlGaN} / \mathrm{GaN}$ heterostructure were specifically prepared. The samples S1 and S2 are the AlGaN/ GaN heterostructures without and with inserting a nominal 2nm-thick $\mathrm{AlN}_{\mathrm{ex}}$ layer at the interface. The $\mathrm{AlN}_{\mathrm{ex}}$ layer growth at a rate of $\sim 3.5 \mathrm{~nm} / \mathrm{min}$ was carried out with our standard recipe, ${ }^{7}$ a TMAl flow of $0.7 \mathrm{ml} / \mathrm{min}(31.5 \mu \mathrm{mol} / \mathrm{min})$ together with a $\mathrm{NH}_{3}$ flow of $21 / \mathrm{min}(2000 \mathrm{sccm})$. For the sample S3, the heterostructure was grown with the aim to obtain a sharp interface. Thus, a low flow rate of TMAl, $0.23 \mathrm{ml} / \mathrm{min}$ $(10.4 \mu \mathrm{mol} / \mathrm{min})$, together with the $\mathrm{NH}_{3}$ flow of $2 \mathrm{l} / \mathrm{min}$ for $13 \mathrm{~s}$ was introduced to the growth zone right after the $\mathrm{GaN}$ growth. Besides, it is worth pointing out that a 200-nm-thick intrinsic $\mathrm{GaN}$ spacer layer grown at $1080^{\circ} \mathrm{C}$ with residual $\mathrm{C}$, $\mathrm{Si}$, and $\mathrm{O}$ concentrations below the SIMS detection limit of $\sim 1 \times 10^{16} \mathrm{~cm}^{-3}$ was used in all the HEMT structures to prevent possible trapping effects and ionized impurity scattering that can adversely affect 2 DEG mobility. ${ }^{12}$ The samples were characterized by mercury-probe capacitance-voltage (CV) measurements to extract the pinch-off voltage. A contactless eddy-current technique and a Lehighton (LEI 1610) contactless mobility system were performed to measure the sheet resistance (Rs) and the 2DEG density and the mobility, respectively. Afterwards, STEM was employed to analyze the interfacial structures of the three samples. Table I summaries the electrical properties of the three HEMT samples. 
TABLE I. Summary of the electrical properties of the samples S1, S2, and S3.

\begin{tabular}{lccc}
\hline \hline HEMT sample & $\mathrm{S} 1$ & $\mathrm{~S} 2$ & $\mathrm{~S} 3$ \\
\hline Interface & No AlNex & AlNex & Sharpened \\
2DEG mobility $\left(\mathrm{cm}^{2} / \mathrm{V} \cdot \mathrm{s}\right)$ & 1669 & 2190 & 2154 \\
2DEG density $\left(\mathrm{cm}^{-2}\right)$ & $6.34 \times 10^{12}$ & $7.8 \times 10^{12}$ & $6.50 \times 10^{12}$ \\
Rs $(\Omega / \mathrm{sq})$ & 612 & 330 & 450 \\
Pinch-off voltage $(\mathrm{V})$ & -3.0 & -4.2 & -3.1 \\
\hline \hline
\end{tabular}

Comparing the samples S1 and S2, it is clear to see that the 2DEG mobility was greatly enhanced from 1669 to $2154 \mathrm{~cm}^{2} / \mathrm{V} \cdot \mathrm{s}$, as expected, with insertion of the $\mathrm{AlN}_{\mathrm{ex}}$ layer. However, the 2DEG density and the pinch-off voltage were also increased, indicating that the thickness and the polarization contributed from the $\mathrm{AlN}_{\mathrm{ex}}$ layer are not negligible. On the other hand, the sample S3 exhibits almost identical electrical properties as sample S1, except for a very high mobility, up to $2140 \mathrm{~cm}^{2} / \mathrm{V} \cdot \mathrm{s}$. This mobility improvement leads to $36 \%$ reduction of Rs, from $612 \Omega /$ sq of S1 to $450 \Omega /$ sq of $S 3$. To rule out the possibility that the difference observed in the mobility might be associated with different levels of dislocation scattering, ${ }^{13}$ high-resolution $\mathrm{x}$-ray diffraction rocking curve measurements were carried out. The crystalline quality of the three samples was found similar. The rocking curves of the GaN (002) and (102) peaks in full width of half maximum for the three HEMT samples are all similar, $\sim 120$ and $\sim 200$ arcsec, respectively. Therefore, in order to clarify the mechanism of the enhanced mobility exhibited from S3, cross-sectional STEM was then performed on the three HEMT samples to access the structural characteristics in the $\mathrm{AlGaN} / \mathrm{GaN}$ interface region. Prior to the analysis, the STEM samples were prepared by standard polishing and argon ion milling procedures. The imaging was performed using the double aberration corrected Linköping Titan $^{3}$ 60-300 (S)TEM equipped with a monochromated high brightness Schottky field emission gun operating at $300 \mathrm{kV}$. Energy dispersive X-ray (EDX) measurements were performed with a Super-X EDX spectrometer and STEM images were acquired using a high angle annular dark field (HAADF) detector.

The cross-sectional STEM-HAADF images and the corresponding EDX line profiles across the AlGaN/GaN interface for the samples S1, S2, and S3 are shown in Fig. 1. Figs. 1(a)-1(c) show the STEM-HAADF images of the samples $\mathrm{S} 1, \mathrm{~S} 2$, and S3, respectively, where the dashed lines and the arrows indicate the EDX probing direction and the interfaces, respectively. As seen in Fig. 1(a), the interface of S1 is obscure, suggesting that the elemental composition across the interface is mixed. In contrast, the sample S2 with the nominal $\mathrm{AlN}_{\mathrm{ex}}$ layer shows a very sharp $\mathrm{AlN}_{\mathrm{ex}} / \mathrm{GaN}$ interface as shown in Fig. 1(b). However, it is clear that the following $\mathrm{AlN}_{\mathrm{ex}} / \mathrm{AlGaN}$ interface is not as sharp as the $\mathrm{AlN}_{\mathrm{ex}} /$ $\mathrm{GaN}$ interface. Besides, the $\mathrm{AlN}_{\mathrm{ex}}$ layer thickness was confirmed to be around $2.5 \mathrm{~nm}$ by taking the second derivative of the Al-K EDX line profile. Furthermore, comparing Fig. 1(c) with Fig. 1(a), the sample S3 apparently exhibits a sharper AlGaN/GaN interface than S1. The interface sharpness is more readily visualized by presenting the Al-K EDX (a)

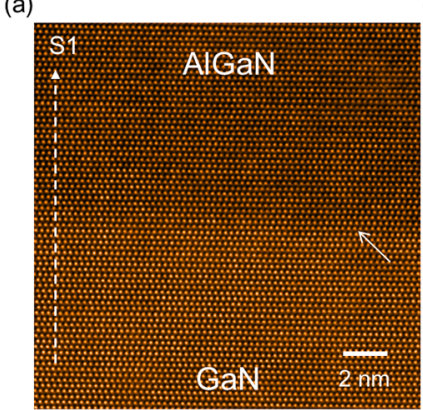

(c)

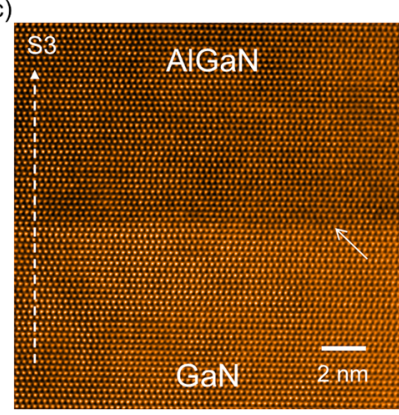

(b)

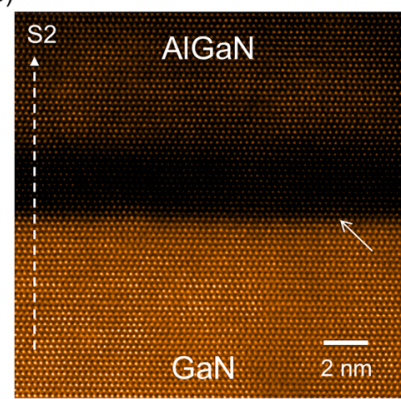

(d)

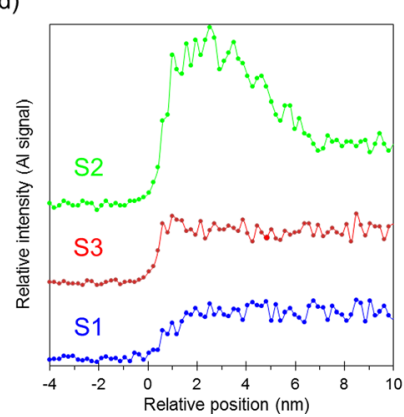

FIG. 1. STEM-HAADF images of the samples (a) S1, (b) S2, and (c) S3, and (d) the EDX spectra of the three samples in the AlGaN/GaN interface region.

line profile across the interface, starting from the GaN layer and into the $\mathrm{AlGaN}$ or $\mathrm{AlN}_{\mathrm{ex}}$ layers. The corresponding EDX spectra of the three samples are showed in Fig. 1(d), with the onset of the interfaces aligned with respect to each other to facilitate comparison. For the samples S1 and S3, though both exhibit the same heterostructure, their Al profiles are quite different as shown. The Al profile from the $\mathrm{GaN}$ to the $\mathrm{AlGaN}$ in $\mathrm{S} 1$ requires $\sim 2.0 \mathrm{~nm}$ to reach the level at the average concentration of the AlGaN layer, while the transition in S3 is comparably abrupt and requires less than $1.0 \mathrm{~nm}$ to the Al level of the AlGaN. We propose that this is the explanation for the recorded mobility difference as seen between the samples S1 and S3. This proposal is further supported by the fact that the high-mobility sample S2 also exhibits an abrupt Al transition at the interface of $\mathrm{AlN}_{\mathrm{ex}} /$ $\mathrm{GaN}$.

In principal, a sharp-interface $\mathrm{AlGaN} / \mathrm{GaN}$ heterostructure would enable better carrier confinement as a result of the abrupt conduction band offset, which is supposed to give rise to the same effect on the 2DEG mobility as the insertion of an $\mathrm{AlN}_{\mathrm{ex}}$ layer into the heterostructure. To confirm this scenario, a multi-carrier mobility-spectrum measurement ${ }^{14}$ was performed in the Lehighton mobility system on the three HEMT samples, the analysis of which can resolve individual carrier mobilities, densities, and their contributions to the conductivity in multi-carrier materials. The mobility spectrum gives an idea of the mobility distributions of various fractions of the free electrons. The analysis result is shown in Fig. 2. The pink, red, and blue spectra represent the mobility distributions of the $90 \%, 50 \%$, and $10 \%$ of the free electrons, respectively. Obviously, S2 and S3 have much higher peak mobility than S1. More importantly, S1 shows a broader mobility distribution than S2 and S3. This evidences that the carrier confinement in sharp-interface S2 and S3 is similar and indeed better than that in S1, consequently 


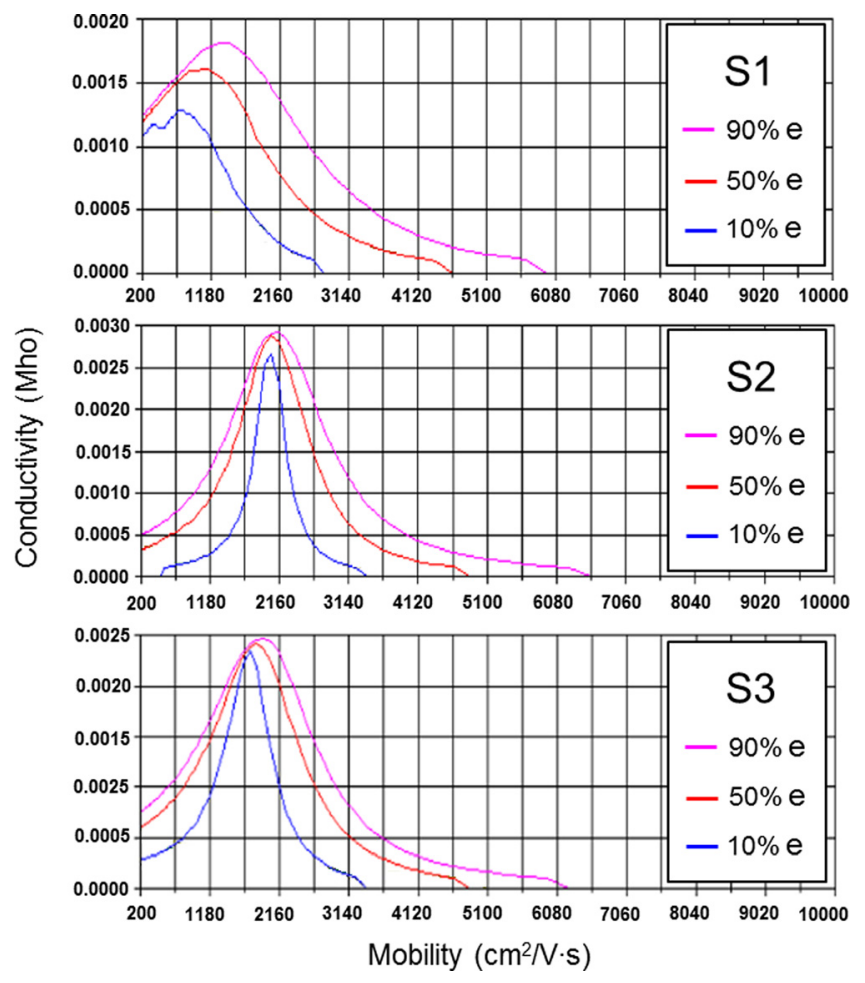

FIG. 2. Multi-carrier mobility distributions of the samples S1, S2, and S3.

leading to higher mobility. We found that this experimental work is in good agreement with a theoretical calculation ${ }^{15}$ predicting that when the width of the $\mathrm{AlGaN} / \mathrm{GaN}$ heterointerface is reduced from $2 \mathrm{~nm}$ to $0.5 \mathrm{~nm}$, the amount of electron gas located in GaN would be considerably increased, so that the 2DEG experiences less alloy disorder and/or interface roughness scattering.

Clearly, the introduction of the low-flow-rate TMAl to the growth zone during the growth transition from the GaN to the $\mathrm{AlGaN}$ was the key process to abruptly raise the $\mathrm{Al}$ content. It is likely that the TMAl flow can surpass the residual $\mathrm{Ga}$ in the gas phase or on the growth surface, thus the $\mathrm{Ga}$ incorporation is suppressed to a certain extent during the interface transition. The influence of the residual $\mathrm{Ga}$ can be also observed on the nominal $\mathrm{AlN}_{\mathrm{ex}}$ layer, because in fact the $\mathrm{AlN}_{\mathrm{ex}}$ layer is a high $\mathrm{Al}$-content $\mathrm{AlGaN}$ layer instead of a pure AlN layer. Such influences from the residual elements could result in a memory effect, which leads to a gradual and diffuse elemental transition at the interface like the AlGaN/

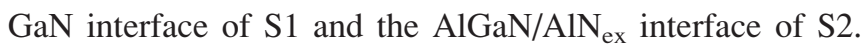
This has been a common phenomenon seen in MOCVD growth processes. Now, the proposed method for interface sharpening seems to be an effective process route.

To establish a reproducible interface sharpening process, another series of HEMT samples containing a 21-nm-thick $\mathrm{Al}_{0.19} \mathrm{Ga}_{0.81} \mathrm{~N} / \mathrm{GaN}$ heterostructure were grown, the interface of which was exposed to a TMAl flow of $0.20 \mathrm{ml} / \mathrm{min}$ $(9.0 \mu \mathrm{mol} / \mathrm{min})$ with a $\mathrm{NH}_{3}$ flow of $21 / \mathrm{min}$ for varying duration before the AlGaN growth. Fig. 3 shows the 2DEG density, the mobility, and the pinch-off voltage as a function of the TMAl flow time, measured from this series of HEMT samples. From Fig. 3(a), no explicit increase of the 2DEG density and the pinch-off voltage was observed until the TMAl flow time reached $30 \mathrm{~s}$, suggesting that no distinct (a)
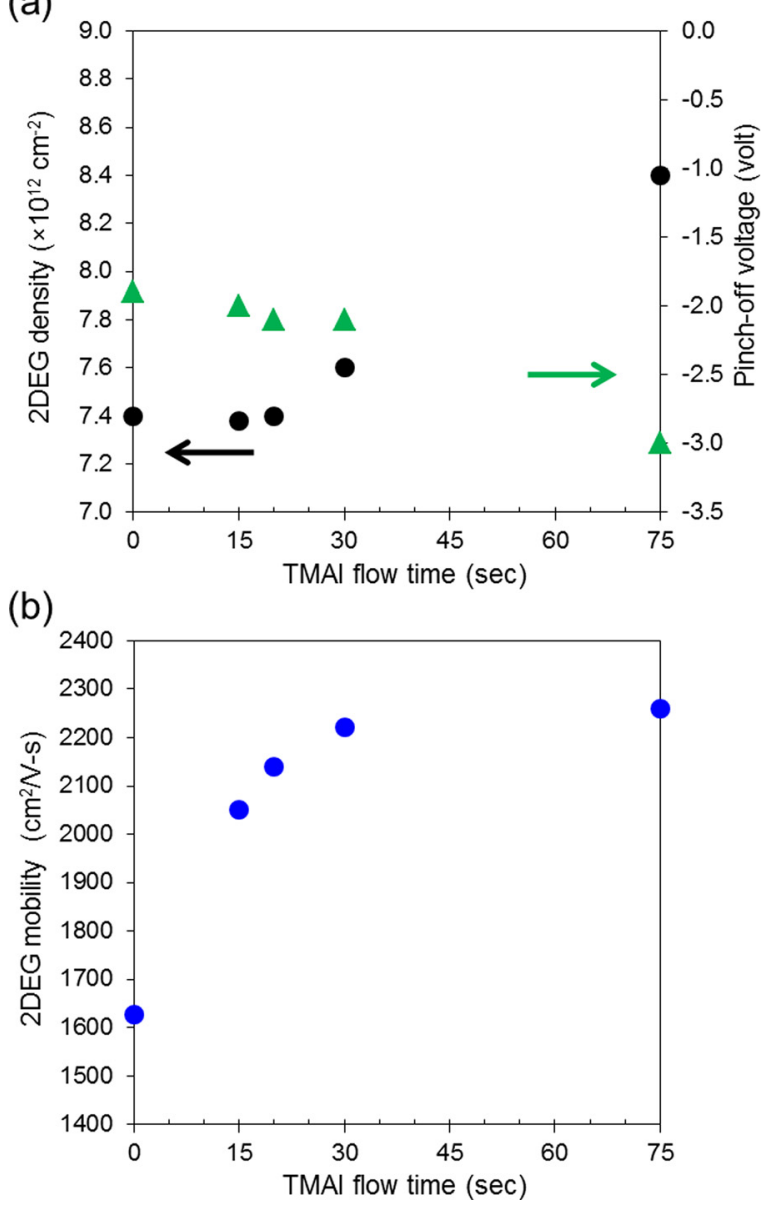

FIG. 3. (a) $2 \mathrm{DEG}$ density and pinch-off voltage as a function of the TMAl flow time and (b) 2DEG mobility as a function of the TMAl flow time.

pure $\mathrm{AlN}$ or high $\mathrm{Al}$-content $\mathrm{AlGaN}$ interlayer was formed at the interface of $\mathrm{AlGaN} / \mathrm{GaN}$ heterostructure when the TMAl flow time of $<30 \mathrm{~s}$ was applied. The slight increase in the 2DEG density and the pinch-off voltage is likely associated with the additional piezoelectric polarization contributed from the abrupt raise of the $\mathrm{Al}$ content at the interface. Moreover, provided that the thickness of AlN growth is linearly proportional to the TMAl flow rate and flow time, the TMAl flow of $0.20 \mathrm{ml} / \mathrm{min}$ for $30 \mathrm{~s}$ would give rise to approximately a 0.5 -nm-thick AlN layer, which is in the same order of a unit cell height of III nitride materials. Thus, the flow time of $<30 \mathrm{~s}$ should not lead to a formation of a single interlayer. However, the mobility was increased considerably from 1630 to $\sim 2050 \mathrm{~cm}^{2} / \mathrm{V}$.s when the TMAl flow time of 15 and $20 \mathrm{~s}$ was applied, and then it saturated around $2250 \mathrm{~cm}^{2} / \mathrm{V} \cdot \mathrm{s}$ when the flow time is $\geq 30 \mathrm{~s}$, as shown in Fig. 3(b). Therefore, it appears that a stable interface sharpening process can be realized when a low-flow-rate TMAl is applied. The optimal flow time would depend on the reactor configuration, the wafer size, and so forth.

Finally, the properties of the sharp-interface $\mathrm{Al}_{\mathrm{x}} \mathrm{Ga}_{1-\mathrm{x}} \mathrm{N} /$ $\mathrm{GaN}$ heterostructures with different $\mathrm{Al}$ contents of the AlGaN were investigated. A series of HEMT samples with three different compositions for a 13-nm AlGaN barrier layer such that $\mathrm{x}=0.20,0.32$, and 0.45 , respectively, were grown using the interface sharpening recipe of the $20 \mathrm{~s}$ TMAl flow time. The 2DEG density was increased from $6.0 \times 10^{12} \mathrm{~cm}^{-2}$ 


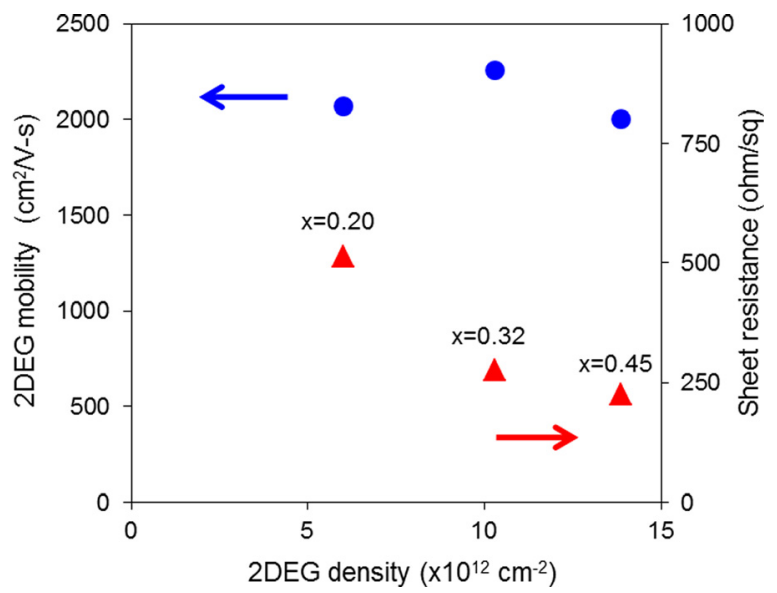

FIG. 4. 2DEG mobility and sheet resistance as a function of the 2DEG density and the corresponding $\mathrm{Al}_{\mathrm{x}} \mathrm{Ga}_{1-\mathrm{x}} \mathrm{N}$ composition.

to $1.4 \times 10^{13} \mathrm{~cm}^{-2}$ as a result of the increased $\mathrm{Al}$ content in the AlGaN barrier. Nevertheless, the 2DEG mobility was very high for all the samples, as shown in Fig. 4. The lowest sheet resistance obtained in the series was $225 \Omega /$ sq for the sample with $\mathrm{x}=0.45$ barrier layer. The highest mobility was $2250 \mathrm{~cm}^{2} / \mathrm{V}$.s for the sample with the 2DEG density of $1.0 \times 10^{13} \mathrm{~cm}^{-2}$. This result highlights the importance of the interface sharpness in the 2DEG transport properties.

Furthermore, since it has been shown that the residual impurities in $\mathrm{AlGaN}$ tend to increase with $\mathrm{Al}$ content, especially when the growth is not performed at a very high temperature, ${ }^{16-18}$ using a sharp interface instead of inserting an AlN exclusion layer in the $\mathrm{AlGaN} / \mathrm{GaN}$ heterostructure thus has a great potential of reducing impurity-related scattering and trapping effects. Both effects could adversely influence the 2DEG properties, especially when the hot electrons are injected or pushed into the AlN or high-Al-content $\mathrm{AlGaN}$ exclusion layer during high-field device operations. ${ }^{9,19,20}$

In summary, a very high 2DEG mobility was achieved in a simple $\mathrm{AlGaN} / \mathrm{GaN}$ heterostructure by the sharpened interface grown in a MOCVD reactor. Beyond this improvement, it is promising for this type of high-mobility heterostructure to have lower contact resistance and to experience less impurity-related scattering and trapping when operating at high-frequency high-field regimes. Additionally, the interface sharpening method can also be applied to enhance the carrier confinement in the growth of other heterostructures such as multi-quantum wells in light emitting diode structures.

The authors would like to acknowledge the funding support from the Swedish Foundation for Strategic Research, the Swedish Research Council, the Kunt and Alice Wallenberg Foundation, and the European FP7 project of High Quality European GaN-Wafer on SiC Substrates for Space Applications (EuSiC).

${ }^{1}$ L. Hsu and W. Walukiewicz, Phys. Rev. B 56, 1520 (1997).

${ }^{2}$ L. Hsu and W. Walukiewicz, J. Appl. Phys. 89, 1783 (2001).

${ }^{3}$ N. Maeda, K. Tsubaki, T. Saitoh, T. Tawara, and N. Kobayashi, Opt. Mater. 23, 211 (2003).

${ }^{4}$ V. M. Polyakov, V. Cimalla, V. Lebedev, K. Köhler, S. Müller, P. Waltereit, and O. Ambacher, Appl. Phys. Lett. 97, 142112 (2010).

${ }^{5}$ R. S. Balmer, K. P. Hilton, K. J. Nash, M. J. Uren, D. J. Wallis, A. Wells, M. Missous, and T. Martin, Phys. Status Solidi C 0, 2331 (2003).

${ }^{6}$ X. Wang, G. Hu, Z. Ma, J. Ran, C. Wang, H. Xiao, J. Tang, J. Li, J. Wang, Y. Zeng, J. Li, and Z. Wang, J. Cryst. Growth 298, 835 (2007).

${ }^{7}$ U. Forsberg, A. Lundskog, A. Kakanakova-Georgieva, R. Ciechonski, and E. Janzén, J. Cryst. Growth 311, 3007 (2009).

${ }^{8}$ R. S. Balmer, K. P. Hilton, K. J. Nash, M. J. Uren, D. J. Wallis, D. Lee, A. Wells, M. Missous, and T. Martin, Semicond. Sci. Technol. 19, L65 (2004).

${ }^{9}$ Y. F. Wu, d. Kapolnek, J. P. Ibbetson, P. Parikh, B. P. Keller, and U. K. Mishra, IEEE Trans. Electron Devices 48, 586 (2001).

${ }^{10}$ J. G. Felbinger, M. Fagerlind, O. Axelsson, N. Rorsman, X. Gao, S. Guo, W. J. Schaff, and L. F. Eastman, IEEE Electron Device Lett. 32, 889 (2011).

${ }^{11}$ M. Fagerlind and N. Rorsman, Phys. Status Solidi C 8, 2204 (2011).

${ }^{12}$ J. T. Chen, U. Forsberg, and E. Janzen, Appl. Phys. Lett. 102, 193506 (2013).

${ }^{13}$ S. W. Kaun, M. H. Wong, U. K. Mishra, and J. S. Speck, Appl. Phys. Lett. 100, 262102 (2012).

${ }^{14}$ D. Nguyen, K. Hogan, A. Blew, and M. Cordes, J. Cryst. Growth 272, 59 (2004).

${ }^{15}$ K. A Mkholyan, J. Silcox, Z. Yu, W. J. Schaff, and L. F. Eastman, J. Appl. Phys. 95, 1843 (2004).

${ }^{16}$ A. Y. Polyakova, M. Shin, J. A. Freitas, M. Skowronski, D. W. Greve, and R. G. Wilson, J. Appl. Phys. 80, 6349 (1996).

${ }^{17}$ G. Parish, S. Keller, S. P. Denbaars, and U. K. Mishra, J. Electron. Mater. 29, 15 (2000).

${ }^{18}$ M. Imura, H. Sugimura, N. Okada, M. Iwaya, S. Kamiyama, H. Amano, I. Akasaki, and A. Bandoh, J. Cryst. Growth 310, 2308 (2008).

${ }^{19}$ L. Fu, H. Lu, D. Chen, R. Zhang, Y. Zheng, T. Chen, K. Wei, and X. Liu, Appl. Phys. Lett. 98, 173508 (2011).

${ }^{20}$ M. Meneghini, A. Stocco, R. Silvestri, G. Meneghesso, and E. Zanoni, Appl. Phys. Lett. 100, 233508 (2012). 